\title{
A Rapid and Precise Diagnostic Method for Detecting the Pinewood Nematode Bursaphelenchus xylophilus by Loop-Mediated Isothermal Amplification
}

\author{
Taisei Kikuchi, Takuya Aikawa, Yuka Oeda, Nurul Karim, and Natsumi Kanzaki
}

First, third, fourth, and fifth authors: Forestry and Forest Products Research Institute, Tsukuba, Ibaraki 305-8687, Japan; and second author:

Tohoku Research Center, Forestry and Forest Products Research Institute, Morioka, Iwate 020-0123, Japan. Accepted for publication 12 August 2009.

\section{ABSTRACT}

Kikuchi, T., Aikawa, T., Oeda, Y., Karim, N., and Kanzaki, N. 2009. A rapid and precise diagnostic method for detecting the pinewood nematode Bursaphelenchus xylophilus by loop-mediated isothermal amplification. Phytopathology 99:1365-1369.

Bursaphelenchus xylophilus is the causal agent of pine wilt disease, which is a major forest disease in Japan, Korea, China, Taiwan, and Portugal. A diagnostic method which is rapid, precise, and simple could greatly help the proper management of this disease. Here, we present a novel detection method using loop-mediated isothermal amplification (LAMP) targeting the internal transcribed spacer region of ribosomal DNA of the nematode. Specificity of the primers and LAMP was confirmed using DNA from various nematode species related to $B$. xylophilus. Our experimental results suggest that LAMP can detect $B$. xylophilus faster and with higher sensitivity than the traditional diagnostic method. Moreover, because it does not require expensive equipment or specialized techniques, this LAMP-based diagnostic method has the potential to be used under field conditions.
The pine wood nematode Bursaphelenchus xylophilus is the causal agent of pine wilt disease, which is a major forest disease in Japan and East Asia. The nematode, which has devastated pine trees and pine forests over the last century, is thought to have been introduced to Japan from North America (4). It subsequently spread to other Asian countries, including Korea, Taiwan, and China. The discovery of B. xylophilus in Portugal in 1999 (9), the first report of this nematode in native conifer stands within the European Union, has increased attention on the disease. Many other parts of the world are also at major risk from the disease (18). In order to manage the disease, by practices ranging from quarantine to field management, a rapid and accurate method of diagnosing B. xylophilus is urgently needed.

Quarantine to prevent introduction of the pathogen is the first crucial step in its management, because it is extremely difficult to control B. xylophilus once introduction has occurred. The longrange spread of $B$. xylophilus occurs as a result of human activity. The nematodes are generally thought to be transported in timber used for producing packaging materials. These factors have led to the development of quarantine regulations that aim to prevent the spread and introduction of the pathogen (4). In order to comply with these regulations, accurate techniques for detecting and identifying B. xylophilus are urgently required.

In the field, an easy diagnostic method is desired. Even in Japan, where the disease was introduced over 100 years ago, the area infected by the disease is still expanding. In infected areas, management of dead trees is important to reduce the next generation of the pathogen and to prevent it spreading to uninfected areas. Easy diagnostic methods have the potential to significantly reduce labor costs and achieve effective management.

The traditional diagnostic method is to isolate nematodes from wood samples using the Baermann funnel technique (16) fol-

Corresponding author: T. Kikuchi; E-mail address: kikuchit@affrc.go.jp

doi:10.1094/PHYTO-99-12-1365

(C) 2009 The American Phytopathological Society lowed by microscopic observation. This method, however, is time consuming and require specialized knowledge about the morphology of the nematode, because it is extremely difficult to distinguish the pathogenic species $B$. xylophilus from nonpathogenic related species (6).

In recent years, molecular-based detection methods for plantparasitic nematodes using polymerase chain reaction (PCR) have been developed and applied worldwide $(12,13)$. Rapid detection of B. xylophilus infections by conventional PCR or real-time PCR has also been reported, and it appears to be possible to use PCR for diagnosis $(1,2,5,8,15)$. The development of portable real-time PCR instruments has increased our ability to apply this approach directly in the field for plant disease diagnostics (14). Although there seems to be no published report of field-based application of portable real-time PCR in the diagnosis of nematode infections. This approach has been taken in European Union countries to detect nematodes in the field by this advanced technology. Despite its good specificity and sensitivity, the requirement of high precision of thermal cycling in PCR prevents this powerful method for being widely used as a routine diagnostic tool.

The recent invention of loop-mediated isothermal amplification (LAMP) provides a new alternative for molecular diagnosis $(10,17)$. LAMP uses a set of four to six primers and a DNA polymerase with strand displacement activity (BstDNA polymerase) to amplify DNA with high specificity under isothermal conditions in $<1 \mathrm{~h}(10)$, without the need for a thermal cycler. LAMP products can be visualized either by gel electrophoresis, as for conventional PCR products, or using a number of other methods which may be suitable for use in the field. For example, the addition of a fluorescent dye to a positive LAMP reaction produces a color change which allows detection with the naked eye (3). Because LAMP does not require an expensive thermal cycler and optical detection equipment, this method clearly holds potential for testing in the field or in under-equipped laboratories (11).

In this study, we present a new method of detecting $B$. xylophilus using the LAMP technique and describe an efficient 
means of extracting DNA from nematodes in a wood sample. These methods are easy and highly sensitive, and require no specialized equipment or techniques.

\section{MATERIALS AND METHODS}

Biological materials. All nematode populations used in this study are maintained at the Forestry and Forest Products Research Institute (FFPRI), Japan (Table 1). Nematodes were cultured on the Botrytis cinerea NF1 strain on potato dextrose agar plates. For the preparation of wood samples, $\approx 15 \mathrm{~g}$ of wood was collected individually from four Pinus densiflora trees using a $1.5-\mathrm{cm}-$ diameter drill in Iwate prefecture in Japan. From each collected wood sample, $10 \mathrm{~g}$ was used for Baermann nematode extraction and the remaining $5 \mathrm{~g}$ was used for LAMP diagnosis.

DNA extraction from nematodes, fungi, and plants. For the specificity test, nematode DNA was prepared from $\approx 10,000$ nematodes which were isolated from culture by the Baermann funnel technique (16) for $3 \mathrm{~h}$ at $23^{\circ} \mathrm{C}$ and washed three times by phosphate-buffered saline-Tween. After the nematodes were lysed by incubation at $55^{\circ} \mathrm{C}$ for $20 \mathrm{~min}$ in $100 \mu \mathrm{l}$ of extraction buffer supplied with the Bursaphelenchus xylophilus detection kit (Nippon Gene), the lysate was extracted once with PCI (phenol/ chloroform/isoamyl alcohol, 25:24:1), followed by ethanol precipitation, and finally dissolved in $50 \mu \mathrm{l}$ of Tris-EDTA buffer ( $\mathrm{pH} 8)$.

Wood samples $(\approx 0.12 \mathrm{~g}$ of wood for each of 10 replicate LAMP reactions) were incubated at $55^{\circ} \mathrm{C}$ for $20 \mathrm{~min}$ in $800 \mu \mathrm{l}$ of extraction buffer, which contains proteinase $\mathrm{K}$ and dithiothreitol, supplied with the B. xylophilus detection kit (Nippon Gene) followed by incubation at $95^{\circ} \mathrm{C}$ for $10 \mathrm{~min}$; then, $2 \mu \mathrm{l}$ was directly used for the LAMP reaction.

Genomic DNA of a fungus (Botrytis cinerea) and plant (Pinus spp.) were prepared using a DNeasy plant kit following the manufacturer's instructions (Qiagen).

TABLE 1. Nematode, fungus, and plant species used to assess specificity of loop-mediated isothermal amplification (LAMP) ${ }^{\mathrm{a}}$

\begin{tabular}{|c|c|c|}
\hline Species & Population or strain & $\begin{array}{c}\text { Amplification by } \\
\text { LAMP }\end{array}$ \\
\hline Bursaphelenchus xylophilus & $\mathrm{Ka} 4$ & + \\
\hline B. xylophilus & $\mathrm{T} 4$ & + \\
\hline B. xylophilus & OKD1 & + \\
\hline B. xylophilus & C14-5 & + \\
\hline B. xylophilus & OK3 & + \\
\hline B. mucronatus & Un1 & - \\
\hline B. doui & No. 204 & - \\
\hline B. doui & No. 217 & - \\
\hline B. luxuriosae & No. 205 & - \\
\hline B. yongensis & No. 209 & - \\
\hline B. clavicauda & No. 211 & - \\
\hline B. sinensis & No. 213 & - \\
\hline B. parvispicularis & No. 216 & - \\
\hline B. poligraphi & No. 225 & - \\
\hline B. okinawaensis & No. 212 & - \\
\hline Bursaphelenchus sp. & No. 210 & - \\
\hline Bursaphelenchus sp. & No. 215 & - \\
\hline Bursaphelenchus sp. & No. 221 & - \\
\hline Bursaphelenchus sp. & No. 224 & - \\
\hline Aphelenchoides stammeri & No. 218 & - \\
\hline A. xylocopae & No. 222 & - \\
\hline Aphelenchoides sp. & No. 201 & - \\
\hline Aphelenchoides sp. & No. 223 & - \\
\hline Ruehmaphelenchus sp. & No. 202 & - \\
\hline Aphelenchus avenae & AaF1 & - \\
\hline Diplogasteroides sp. & NKd & - \\
\hline Botrytis cinerea (fungus) & NF1 & - \\
\hline Pinus thunbergii & $\ldots$ & - \\
\hline P. densiflora & $\ldots$ & - \\
\hline
\end{tabular}

a LAMP specifically detected Bursaphelenchus xylophilus.
Primer design. The specific primers for the LAMP reaction were designed using the PrimerExplorer V4 software program (http://primerexplorer.jp). Various genome regions including ITS of rDNA, beta-actin gene, elongation factor 1 alpha gene, and beta-tubulin gene were amplified from $B$. xylophilus and $B$. mucronatus using primers designed from the Expressed Sequence Tag data of these two nematode species (7). Amplified products were sequenced and aligned to compare sequences between the two species. The DNA regions demonstrating greatest dissimilarities were selected for designing B. xylophilus-specific LAMP primers (Fig. 1; see Results). These sequences were submitted to DDBJ with accession nos. AB500146 to AB500156.

LAMP reaction. The LAMP reaction was performed according to the method described by Notomi et al. (10) with the Loopamp DNA amplification kit (Eiken Chemical). Briefly, the reaction was performed in $25 \mu \mathrm{l}$ of reaction mixture containing $2 \mu \mathrm{l}$ of extracted DNA solution, 5 pmol each of F3 and B3 primers, 40 pmol each of FIP and BIP primers, 20 pmol of LoopF primer, $12.5 \mu \mathrm{l}$ of $2 \times$ reaction mix, $1 \mu \mathrm{l}$ of BstDNA polymerase, and $1 \mu \mathrm{l}$ of fluorescent detection reagent (Eiken Chemical). The reaction mixture was incubated at $63^{\circ} \mathrm{C}$ for 60 to $120 \mathrm{~min}$ and terminated by incubation at $80^{\circ} \mathrm{C}$ for 2 min. LAMP amplicons were detected by color changes of the reaction solution under UV light (Fig. 2).

Lateral-flow strip detection. To evaluate amplified products, we used a probe-based detection system. The 5'-biotinylated form of the FIP primer was used for the LAMP reaction. After the LAMP reaction, $10 \mu \mathrm{l}$ of FITC-labeled probe $(10 \mathrm{pmol} / \mu \mathrm{l}) \mathrm{de}$ signed to hybridize to an internal region of the target sequence (Table 2) was added to the reaction mixture and incubated at $95^{\circ} \mathrm{C}$ for $5 \mathrm{~min}$, then slowly cooled to $25^{\circ} \mathrm{C}$. The reaction mixture was diluted with $100 \mu$ l of running buffer (phosphate-buffered saline $+3 \%$ Tween) and applied directly to HybriDetect strips (Milenia Biotec, Germany) according to the manufacturer's instructions. HybriDetect strips detect fragments containing both biotin and FITC resulting from specific amplification. In contrast, when nonspecific amplification occurs, no signal can be observed at the detection line.

Sensitivity test. For the sensitivity test, a plasmid DNA containing the target sequence was made using the pGEM-T Easy System (Promega). The plasmid was transformed and amplified in Escherichia coli DH5 $\alpha$ using standard procedures. After plasmid purification, a dilution series was made and used for calibration. A real-time PCR system was used to monitor the kinetics of LAMP amplification. The LAMP reaction was performed as described above with $2.5 \mu$ of $10 \times$ SYBR Green (Roche) and $0.4 \mu$ l of ROX (Takara) instead of fluorescent detection reagent at $63^{\circ} \mathrm{C}$ for 120 min using the StepOnePlus Real-time PCR System (Applied Biosystems). Amplification was monitored by SYBR Green florescence every $1 \mathrm{~min}$ during the reaction.

TaqMan-based real-time PCR. Real-time PCR with a TaqMan probe was conducted as described previously (1). Primers BXF (5'-GATGATGCGATTGGTGACT-3') and BXR (5'-AACGACGCGAATCGAACC-3') and probe BXT (5'-CGGTTGCCGCGCATGATGG-3') labeled with FAM and TAMRA were used to detect the B. xylophilus-specific DNA. Reaction was performed in $50 \mu \mathrm{l}$ consisting of $25 \mu \mathrm{l}$ of $2 \times$ FastStart TaqMan Probe Master (ROX) (Roche), $400 \mathrm{nM}$ each primer, $200 \mathrm{nM}$ BXT probe, and an appropriate DNA solution. The StepOnePlus (Applied Biosystems) was used for amplification and fluorescence measurement at each temperature step and cycle during the reaction. Thermal cycling conditions consisted of $10 \mathrm{~min}$ at $95^{\circ} \mathrm{C}$ followed by 40 cycles of $15 \mathrm{~s}$ at $95^{\circ} \mathrm{C}$ and $45 \mathrm{~s}$ at $60^{\circ} \mathrm{C}$.

\section{RESULTS}

Primer design. LAMP primer sets were designed to the ITS of rDNA, $\beta$-actin, elongation factor $1 \alpha$, and $\beta$-tubulin genes using 
LAMP primer design software (PrimerExpress). Primers were selected based on the sequence dissimilarity between $B$. xylophilus and B. mucronatus at the primer positions. Primer sets selected were then evaluated for amplification efficiency and generation of false positives (data not shown). A primer set was selected that amplifies the ITS region of rDNA with high efficiency and a low false-positive rate (Fig. 1). This region (Fig. 1 , between primers $\mathrm{F} 3$ and B3) showed 20\% dissimilarity (i.e., 190 in 239 bp is identical) between B. xylophilus and B. mucronatus.

Specificity test. To examine the specificity of the LAMP detection method, we used DNA extracted from several nematode species of the genera Bursaphelenchus, Aphelenchoides, Ruehmaphelenchus, Diplogasteroides, and Aphelenchus (Table 1). We used five distinct populations of B. xylophilus and 20 other nematode populations. Amplifications were detected by color changes of the reaction solution in all B. xylophilus populations (Fig. 2). In contrast, no amplification was observed from other nematode species, including a closely related species, $B$. mucronatus, which is difficult to distinguish from B. xylophilus by its morphological characteristics (Table 1). Furthermore, no amplification was detected from DNA of $B$. cinerea, a fungus that was used as nematode food during cultivation, and two Pinus spp. Amplified products were evaluated by probe hybridization to the inner part of the target sequence using lateral flow strips. All amplified products from $B$. xylophilus showed positive signals at the detection lines of the strips, indicating that the products were not the result of nonspecific amplifications (Fig. 2).

Sensitivity test. The real-time kinetics of the LAMP reaction were studied by monitoring fluorescence as described in Materials and Methods. In preliminary examinations, the LAMP reaction was performed at different temperatures of 60, 63, and
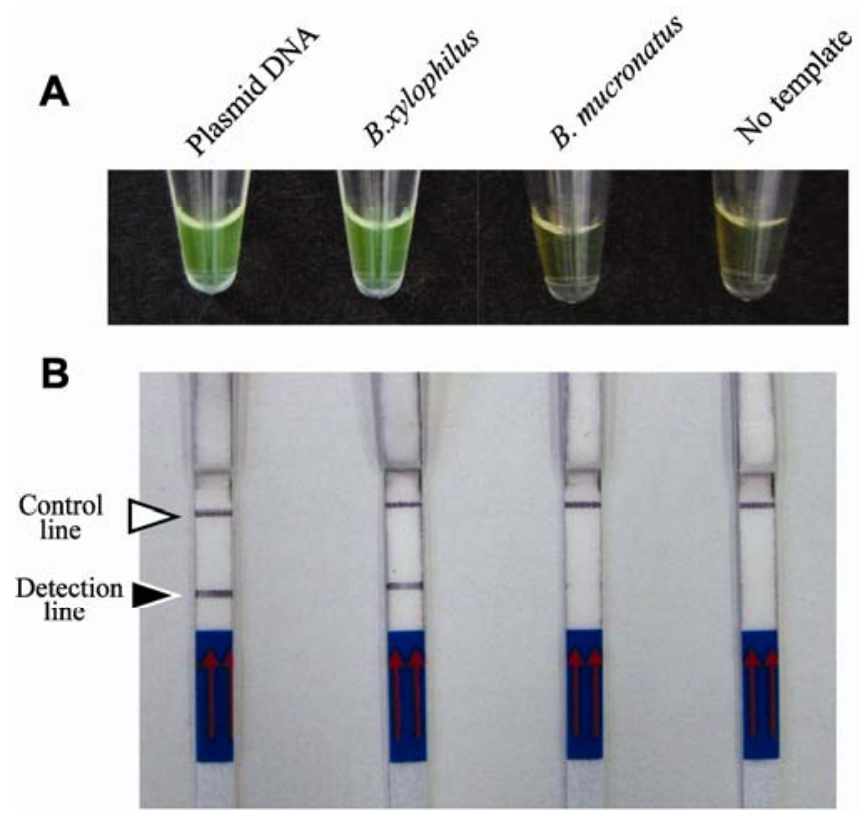

Fig. 2. Visual inspection and lateral-flow strips used for the detection of loopmediated isothermal amplification (LAMP) products. A, LAMP products were visualized by fluorescent detection reagent and UV light. B, FAM-labeled probe was hybridized to an inner part of the target DNA and detected by lateral-flow strips. LAMP reaction was carried out using internal transcribed spacer primers in the presence of plasmid DNA containing the target sequence (positive control) and genomic DNA of Bursaphelenchus xylophilus and B. mucronatus, and in the absence of template DNA (negative control). For lateral-flow strip detection, biotin-labeled FIP primer was used for the LAMP reaction.

A
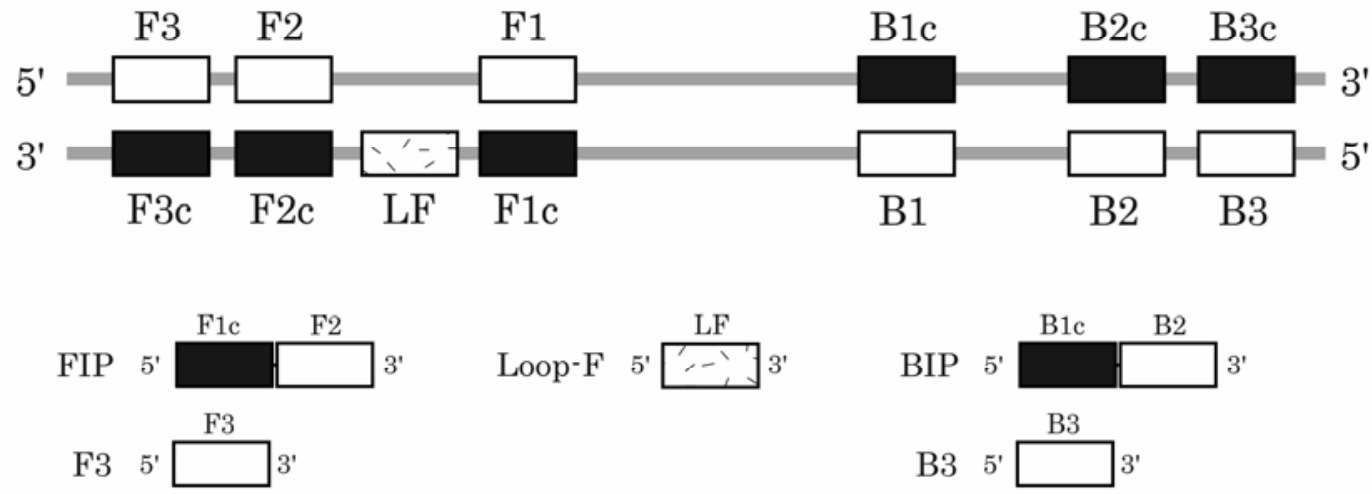

B

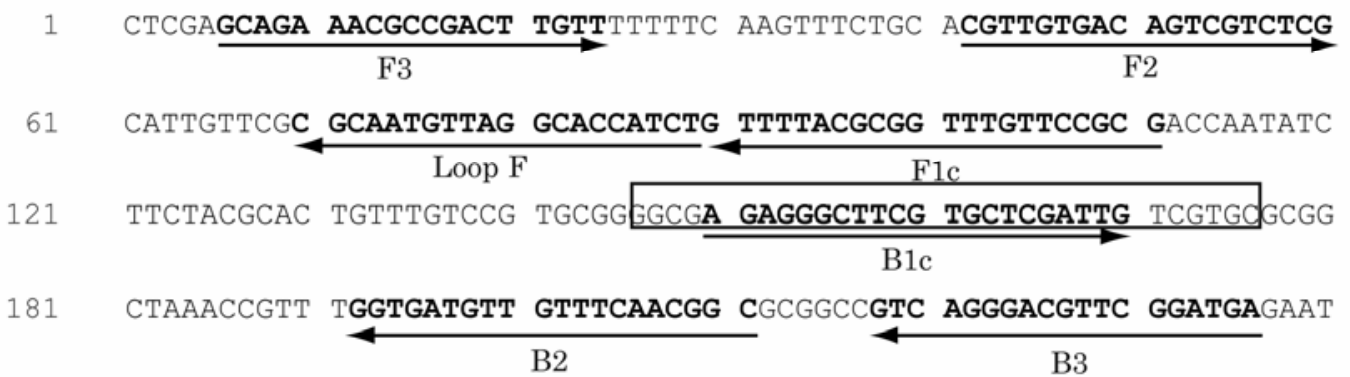

Fig. 1. A, Schematic representation of double-strand target DNA and loop-mediated isothermal amplification inner (FIP and BIP), outer (F3 and B3), and inner loop (Loop-F) primers. The FIP (BIP) primer consists of F1c (B1c) and F2 (B2). B, Nucleotide sequence of Bursaphelenchus xylophilus rDNA internal transcribed spacer region. The underlined letters indicate the sequences used for primers. The box indicates the position of the probe used for lateral-flow strip detection. 
$65^{\circ} \mathrm{C}$, and the reaction at $63^{\circ} \mathrm{C}$ was the most productive (Fig. 3A). Thereafter, LAMP was carried out at $63^{\circ} \mathrm{C}$ for $120 \mathrm{~min}$. Sensitivity of the LAMP assay for detection of B. xylophilus DNA was determined by testing serial 10-fold dilutions of the plasmid DNA carrying the target sequence. Positive signals were successfully detected in all eight independent reactions containing $>10$ copies of the plasmid DNA within 60 min (Fig. 3B). Amplification results using one copy of the plasmid DNA were unstable. Sometimes (three trials), amplification could be observed at the later phase of the reaction (after $90 \mathrm{~min}$ ) but, at other times (five trials), no amplification was observed even after $120 \mathrm{~min}$. These results suggested that the LAMP assay had a detection limit of $\approx 10$ copies of target DNA with a reaction time of $60 \mathrm{~min}$.

In addition, the nematode extract was also used to assess sensitivity. One adult female was lysed in $800 \mu \mathrm{l}$ of extract buffer and serial 10-fold dilutions of the lysate $(2,0.2,0.02$, and $0.002 \mu \mathrm{l})$ were directly used as templates. Amplification products could be detected when at least $0.02 \mu \mathrm{l}$ of the lysate was in the reaction mixture (that is, $2.5 \times 10^{-5}$ of a nematode in the reaction mixture) (Fig. 3C). No amplification was observed in any "notemplate" controls.

The comparative sensitivities of the LAMP and TaqMan-based real-time PCR assays revealed that the LAMP assay was 10-fold more sensitive than the real-time PCR assay, which has a detection limit of 100 copies of the target DNA (Table 2).

TABLE 2. Comparative sensitivities of the loop-mediated isothermal amplification (LAMP) and TaqMan-based real-time polymerase chain reaction (PCR) assay systems for detection of the internal transcribed spacer (ITS) region of ribosomal DNA of Bursaphelenchus xylophilus

\begin{tabular}{lcc}
\hline Template & LAMP & PCR $^{\mathrm{a}}$ \\
\hline Plasmid DNA (copies) & $8 / 8$ & \\
$1.00 \mathrm{E}+05$ & $8 / 8$ & $4 / 4$ \\
$1.00 \mathrm{E}+04$ & $8 / 8$ & $4 / 4$ \\
$1.00 \mathrm{E}+03$ & $8 / 8$ & $4 / 4$ \\
100 & $8 / 8$ & $4 / 4$ \\
10 & $5 / 8$ & $1 / 3^{*}$ \\
1 & $8 / 8$ & $1 / 3^{*}$ \\
Nematode extract $(\mu \mathrm{l})$ & $8 / 8$ & \\
2 & $8 / 8$ & $4 / 4$ \\
0.2 & $2 / 8$ & $4 / 4$ \\
0.02 & $0 / 8$ & $0 / 4$ \\
0.002 & & $0 / 8^{*}$ \\
No template &
\end{tabular}

a Asterisk (*) indicates that a small, nonspecific signal was observed at the later stage of PCR reaction.
Detection of B. xylophilus in wood samples. From a more practical viewpoint, we tested the LAMP method using pine wood samples infected by $B$. xylophilus. The presence of $B$. xylophilus in wood samples was confirmed by the Baermann funnel technique and microscopic observation. DNA was extracted from $\approx 0.12 \mathrm{~g}$ of wood sample using the easy extraction method (see Materials and Methods) and was used as a template for LAMP reactions. For each of the 10 replicate LAMP reactions, wood samples were prepared separately. The LAMP reaction was performed at $63^{\circ} \mathrm{C}$ for $60 \mathrm{~min}$ and amplification was judged by color change of the reaction solution. Amplification was observed in all reactions of wood samples containing nematodes, whereas no amplification was observed from the wood samples without nematodes (Table 3).

\section{DISCUSSION}

In this report, we presented a rapid, precise, and simple diagnostic method to detect B. xylophilus using the LAMP technique, which is easier and faster than the traditional diagnostic method. The traditional Baermann funnel method followed by microscopic observation is time-consuming and requires specialized knowledge about the morphology of nematodes, thus significantly decreasing the efficiency of pest management. In contrast, our method is simple and requires no special techniques or knowledge and, therefore, has the potential to greatly improve pest management.

The primers used for the LAMP amplification specifically detected $B$. xylophilus. Generally, dead pine wood contains many species of nematode, including B. mucronatus, B. sinensis, and Diplogaster spp. Sometimes, nematodes that show similar morphology with B. xylophilus were isolated, leading to a misdiagnosis of the disease. However, the experimental results provided in this study (Fig. 2; Table 2) clearly indicated that the

TABLE 3. Detection of Bursaphelenchus xylophilus in wood sample using loop-mediated isothermal amplification

\begin{tabular}{lccc}
\hline Sample name & $\begin{array}{c}\text { No. of nematodes } \\
\text { per 10 } \text { g of wood }^{\mathrm{a}}\end{array}$ & $\begin{array}{c}\text { Detection } \\
\text { rate }(\%)\end{array}$ \\
\hline N-306up & 3,460 & $10 / 10$ & 100 \\
Oku-1un & 19 & $10 / 10$ & 100 \\
N-133up & 1 & $6 / 10$ & 60 \\
Tamayama & bettion/trial & $0 / 10$ & 0 \\
\hline
\end{tabular}

a Nematode numbers were counted after Berman isolation for $24 \mathrm{~h}$.

b Wood samples were collected from a dead tree in an uninfected area.
A

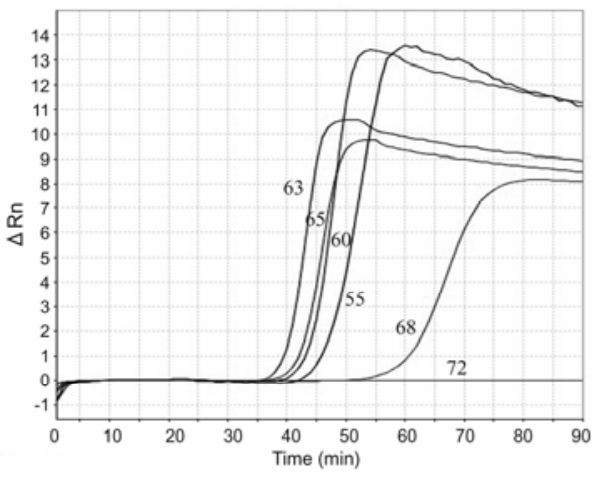

B

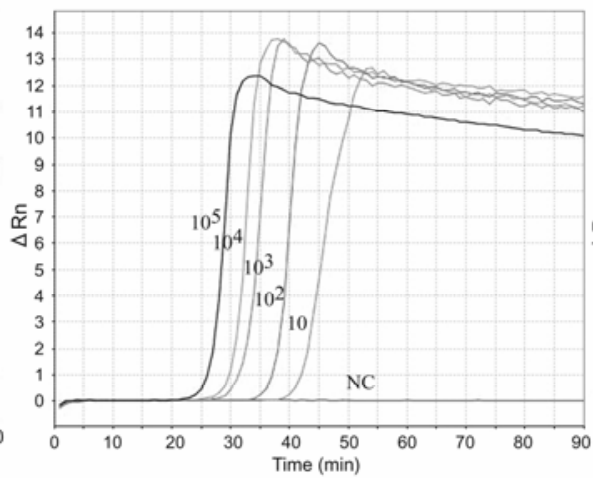

C

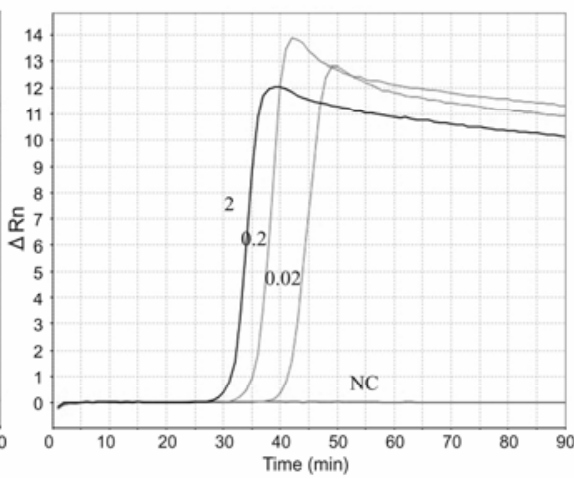

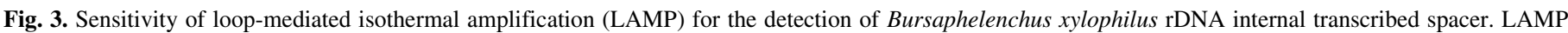

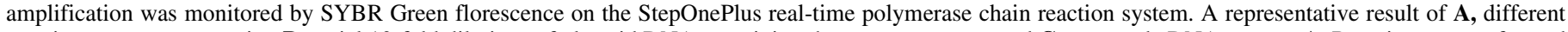

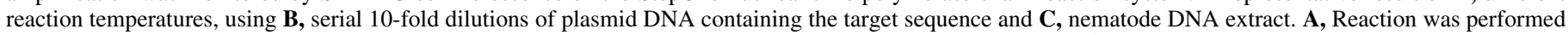

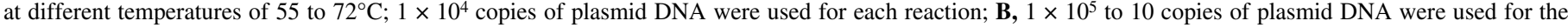

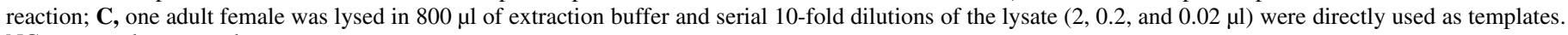
$\mathrm{NC}$ : no-template control 
LAMP method diminished the possibility of this occurring due to the specificity of the primers. One noteworthy observation is that B. xylophilus and B. mucronatus, two very closely related nematodes (in terms of genetic and morphological characteristics), can be distinguished based on the LAMP results (Fig. 2). Moreover, the traditional method cannot make a diagnosis when there is no adult (both male and female) among the isolated nematodes because other stages such as larvae and eggs do not have sufficient morphological specificity to enable identification. In contrast, the LAMP-based diagnosis method can detect B. xylophilus regardless of the life stage of the nematode.

LAMP could reliably detect 10 copies or more of target sequences. More practically, amplification was observed from a small portion of a female nematode $\left(2.5 \times 10^{-5}\right.$ nematodes) (Table 1; Fig. 3C). This sensitivity is relatively high compared with the traditional method or other PCR-based detection methods, indicating that LAMP has good potential to detect hidden infections.

In this study, we also presented an easy method to extract DNA from nematodes in wood samples without nematode isolation. An attempt to detect nematode DNA in wood samples was reported previously (15), using the cetyltrimethylammonium bromide method to extract nematode DNA directly from the wood sample. The method appeared to work well but was extremely laborious and time consuming and had the risk of losing DNA because the wood samples needed to be ground under liquid nitrogen and to undergo several purification processes, including phenol extraction and ethanol precipitation. In contrast, our method does not require any grinding or purification process, can be conducted in one tube, and takes only $20 \mathrm{~min}$. The strength of this method is presumed to lie in the use of appropriate solution to degrade nematode cuticle efficiently and the nonrequirement of a grinding procedure, which avoids PCR inhibitors coming from wood materials. Thus, this method has great potential to reduce the time and cost of diagnosing the disease.

Compared with PCR, LAMP has the advantages of reaction simplicity and detection sensitivity. LAMP does not require complicated thermal cycling steps or expensive thermal cycling machines but only an isothermal reaction for a relatively short time. Another useful feature of LAMP lies in the opportunity for detecting a positive reaction by a color change of the reaction solution which can be judged by the naked eye. Therefore, LAMP can be used as a highly sensitive DNA amplification method in various places, from in the field to local forestry offices, without the cost and environmental constraints prohibiting PCR and other molecular biology techniques. In this study, LAMP-based amplification of DNA has been coupled with a very efficient DNA extraction method, and both LAMP reactions and DNA extraction can be achieved using very simplified equipments such as a temperature controller (water bath), indicating the possibility that the diagnostic method has the potential to be used for on-site detection of nematodes. The LAMP-based diagnostic method described and developed in this study may constitute a turning point in the management of pine wilt disease caused by B. xylophilus.

\section{ACKNOWLEDGMENTS}

This work was partly supported by research grant no. 200704 of FFPRI. N. Karim is supported by the Japan Society for the Promotion of
Science (JSPS) Postdoctoral Fellowship for foreign researchers. T. Kikuchi has been funded by the Japanese Ministry of Education, Science, Sports and Culture, Grant-in-Aid for Encouragement of Young Scientists (B) 18780032 . We thank Y. Ichihara, FFPRI, for assisting with the collection of wood samples from dead pine trees.

\section{LITERATURE CITED}

1. Cao, A. X., Liu, X. Z., Zhu, S. F., and Lu, B. S. 2005. Detection of the pinewood nematode, Bursaphelenchus xylophilus, using a real-time polymerase chain reaction assay. Phytopathology 95:566-571.

2. Francois, C., Castagnone, C., Boonham, N., Tomlinson, J., Lawson, R., Hockland, S., Quill, J., Vieira, P., Mota, M., and Castagnone-Sereno, P. 2007. Satellite DNA as a target for TaqMan real-time PCR detection of the pinewood nematode, Bursaphelenchus xylophilus. Mol. Plant Pathol. 8:803-809

3. Iwamoto, T., Sonobe, T., and Hayashi, K. 2003. Loop-mediated isothermal amplification for direct detection of Mycobacterium tuberculosis complex, $M$. avium, and $M$. intracellulare in sputum samples. J. Clin. Microbiol. 41:2616-2622.

4. Jones, J. T., Moens, M., Mota, M., Li, H., and Kikuchi, T. 2008. Bursaphelenchus xylophilus: Opportunities in comparative genomics and molecular host-parasite interactions. Mol. Plant Pathol. 9:357-368.

5. Kang, J. S. K. 2004. Development of an efficient PCR-based diagnosis protocol for the identification of the pinewood nematode, Bursaphelenchus xylophilus (Nematoda: Aphelenchoididae). Nematology 6:279.

6. Kanzaki, N. 2008. Taxonomy and Systematics of the Nematode Genus Bursaphelenchus (Nematoda: Parasitaphelenchidae). In: Pine Wilt Disease. B. G. Zhao, K. Futai, J. Sutherland, and Y. Takeuchi, eds. Springer, Japan.

7. Kikuchi, T., Aikawa, T., Kosaka, H., Pritchard, L., Ogura, N., and Jones, J. T. 2007. Expressed sequence tag (EST) analysis of the pine wood nematode Bursaphelenchus xylophilus and B. mucronatus. Mol. Biochem. Parasitol. 155:9-17.

8. Leal, I., Green, M., Allen, E., Humble, L., and Rott, M. 2005. An effective PCR-based diagnostic method for the detection of Bursaphelenchus xylophilus (Nematoda: Aphelenchoididae) in wood samples from lodgepole pine. Nematology 7:833-842.

9. Mota, M. M., Braasch, H., Bravo, M.A., Penas, A.C., Burgermeister, W., Metge, K., and Sousa, E. 1999. First report of Bursaphelenchus xylophilus in Portugal and in Europe. Nematology 7:727-734.

10. Notomi, T., Okayama, H., Masubuchi, H., Yonekawa, T., Watanabe, K., Amino, N., and Hase, T. 2000. Loop-mediated isothermal amplification of DNA. Nucleic Acids Res. 28:e63.

11. Okuda, M., Matsumoto, M., Tanaka, Y., Subandiyah, S., and Iwanami, T. 2005. Characterization of the tuf B-sec E-nus G-rpl KAJL-rpo B gene cluster of the citrus greening organism and detection by loop-mediated isothermal amplification. Plant Dis. 89:705-711.

12. Perry, R. N., Subbotin, S. A., and Moens, M. 2008. Molecular diagnostics of plant-parasitic nematodes. In: Biotechnology and Plant Disease Management. Z. K. Punja, S. H. De Boer, and H. Sanfacon, eds. CABI, UK.

13. Powers, T. 2004. Nematode molecular diagnostics: From bands to barcodes. Annu. Rev. Phytopathol. 42:367-383.

14. Schaad, N. W., and Frederick, R. D. 2002. Real-time PCR and its application for rapid plant disease diagnostics. Can. J. Plant Pathol. 24:250258.

15. Takeuchi, Y., Kanzaki, N., and Futai, K. 2005. A nested PCR-based method for detecting the pine wood nematode, Bursaphelenchus xylophilus, from pine wood. Nematology 7:775.

16. Thorne, G. 1961. Principles of Nematology. McGraw-Hill, New York.

17. Tomita, N., Mori, Y., Kanda, H., and Notomi, T. 2008. Loop-mediated isothermal amplification (LAMP) of gene sequences and simple visual detection of products. Nat. Protocols 3:877-882.

18. Vieira, P. R., and Mota, M. M. 2008. Pine Wilt Disease: A Worldwide Threat to Forest Ecosystems. Springer Verlag, The Netherlands. 